\title{
Heat Illness - Recent Developments
}

\author{
RJ Booker, MCM Bricknell
}

\begin{abstract}
This paper reviews the literature concerning exertional heat illness in soldiers. It focuses on developments since Bricknell's two part paper "Heat Illness - A Review of Military Experience" published in this journal in 1996. Recent advances in the understanding of risk factors, pathophysiology and treatment are discussed with a view to reducing the already low incidence of heat illness within the Armed Forces.
\end{abstract}

\section{Introduction}

The current Defence Council Instruction (DCI) concerning heat illness (HI) records that 516 British Armed Forces personnel were admitted to hospital suffering from $\mathrm{HI}$ during the five-year period 1993-1997 (1). Army policy has successfully reduced the incidence of HI mainly through restricting training at higher environmental temperatures (2). Cases still occur at moderate environmental temperatures $(3,4,5)$ and although most are not serious, an average of one death per year draws intense media criticism (6) and renders complacency impossible (4). This paper summarises current knowledge of $\mathrm{HI}$, focusing on developments since the 1996 publication of Lt Col Bricknell's two-part review $(7,8)$. Following a brief overview of the subject, recent developments in the knowledge of risk factors, pathophysiology and interventions applicable to HI will be discussed to suggest future avenues of research.

\section{Overview of Heat Illness}

Any personnel incapacitated due to a rise in body temperature are considered as suffering 2Lt RJ Booker RAMC

Medical Student

St. Bartholomew's and

The Royal London

School of Medicine.

Email: ha8010@qmul.ac.uk

Lt Col MCM Bricknell RAMC

DM MMedSci

MRCGP MFOM

DFPHM DRCOG

DMCC DFFP

Commanding Officer,

22 Field Hospital,

Aldershot, Hants. weakness, malaise and headache wi altered mental status (11). Heat stroke is the hot conditions (8). Heat cramps are painful muscle contractions thought to result from a hyponatraemic electrolyte imbalance (11). Heat exhaustion is indicated by feelings of severest form of HI. Traditionally diagnosed by a core temperature $>40^{\circ} \mathrm{C}$, it is better defined by severe mental dysfunction due to exercise and/or heat stress, regardless of body temperature. There may be prodromal signs synonymous with heat exhaustion, but in $80 \%$ of cases the onset is sudden (12).

There are two pathways to heat stroke. Classical heat stroke occurs in older people during heat waves and originates from loss of efficient temperature control mechanisms (11). Exertional heat stroke occurs when heat is generated by muscular effort at a greater rate than can be dissipated by the body (13). When the normal physiological routes of heat loss (sweating and cutaneous vasodilatation) are overwhelmed, splanchnic vasoconstriction is lost (12). This heralds a fall in blood pressure and impending collapse. This paper is concerned with exertional heat stroke (HS), as this is the type of most concern to the Army Medical Services.

Successive DCIs have sought to reduce HI by limiting exercise according to environmental heat load as measured by the Wet Bulb Globe Temperature Index (WBGT) (1). Experimental validity for these restrictions has been provided by Bricknell (9). Epidemiological analyses of HI cases have always shown seasonal peaks during the warmest months of the year $(13,14)$. However, fatal cases of HS have occurred regardless of environmental heat load $(3,5)$. Indeed, a recent American case series by Kark et al found the peak attack rate for $\mathrm{HI}$ was between 0700-0900 hours. It was between these hours when maximum intensity training was permitted on the grounds that recruits were exposed to the lowest environmental heat load (14). This confirms that the source of the heat causing $\mathrm{HI}$ is endogenous (5). Such observations have changed the focus on $\mathrm{HI}$ prevention towards individual factors. It has been hypothesised that a minority of $\mathrm{HI}$ cases may be linked to congenital disease such as cystic fibrosis (15) or malignant hyperthermia (16).

\section{Method}

In April 2000, Medline searches were conducted for the period 1996-2000 for the following $\mathrm{MeSH}$ headings: "Heat Stress Disorders", "Heat Stroke" and "Heat Exhaustion", producing 355 hits. This search was repeated for the period 1966-2000 and produced 1250 hits, which was reduced to 
254 by limiting to American Institute of Medicine (AIM) publications. Searches were not limited to humans or by age. Additional searches were conducted on the Web of Science Citation Index, which did not yield any additional references. The 609 papers had their abstracts scanned to ascertain relevance. Animal studies were included to provide insights into human physiology and possible new treatments. Reference lists were cross-checked. In December 2001, another Medline search was conducted for the period 2000-2001 for the same three MeSH headings: "Heat Stress Disorders", "Heat Stroke" and "Heat Exhaustion", which captured 190 recently published papers for perusal.

\section{Results}

\section{Risk Factors}

Group risk factors have been outlined in previous research: lack of sleep, food or water, inadequate acclimatisation, environmental heat and the wearing of protective clothing (8). Individual risk factors are the main consideration of this paper: low physical fitness, obesity, past history of HI, sickle cell trait (SCT), various myopathies, dehydration and carriage of the cystic fibrosis gene. These and other previously identified risk factors are listed in Table 1.

Table 1. Individual Risk Factors for Heat Illness

\begin{tabular}{|c|c|}
\hline & Low physical fitness \\
\hline & Obesity \\
\hline & Previous history of HI \\
\hline & Sickle Cell Trait \\
\hline & ? Congenital muscle pathology \\
\hline & Dehydration \\
\hline & ? Carriage of the cystic fibrosis gene \\
\hline & Participation in high-stress training \\
\hline & Sweat gland dysfunction \\
\hline & Concurrent illness \\
\hline & Increasing age \\
\hline & Deep burns \\
\hline & Alcohol \\
\hline & $\begin{array}{l}\text { Therapeutic Drugs: } \\
\text { ß-blockers, methyldopa, diuretics, MAO } \\
\text { inhibitors, laxatives, tricyclic antidepressants, } \\
\text { anticholinergics (atropine), phenothiazines, } \\
\text { antihistamines, vasoconstrictors }\end{array}$ \\
\hline & $\begin{array}{l}\text { Drugs of Abuse: } \\
\text { Amphetamines, cannabinoids, cocaine, opiates, } \\
\text { LSD }\end{array}$ \\
\hline
\end{tabular}

Obesity and lack of physical fitness independently predict risk of suffering HI. Gardner et al studied United States Marine Corps (USMC) recruits and identified a high risk of $\mathrm{HI}$ in those with body mass index (BMI) $\geq 22$ and 1.5 mile run time $\geq 12 \mathrm{~min}$ (10). This high risk population was eight times more likely ( $95 \%$ CI, 3.8-19) to suffer an episode of $\mathrm{HI}$ during basic training than those with a lower BMI $(<22)$ and faster run time $(<10 \mathrm{~min})$. The $20 \%$ of recruits classified as high risk provided nearly half $(47 \%)$ of the total $\mathrm{HI}$ cases occurring in the 12 week basic training cycle. The authors noted that the parameters of BMI and 1.5 mile run time are easily screened for at the outset of training. Thus, implementing effective intervention for this high-risk group could potentially halve the incidence of HI. Suggested interventions included training those at higher risk in the cooler months and slower conditioning schedules.

Recurrence of $\mathrm{HI}$ in seemingly susceptible personnel has been reported infrequently (17) with occasional fatalities (6). Research is contradictory with regard to the duration of heat intolerance following an episode of HI. All nine Israeli soldiers tested 2-5 years after an episode of HS still exhibited heat intolerance compared to matched controls (17). Ten American HS subjects were tested 61 days post HS; nine had regained heat tolerance, one remained heat intolerant, was re-tested twice and eventually reclassified heat tolerant 11.5 months post HS (18). A 1999 paper by Chung et al investigated soldiers nine months after an episode of HS (19). Subjects exhibited an increase in absolute serum cytokine concentrations and a poorer thermo-regulatory response to heat stress in terms of core temperature and sweat loss compared to healthy controls. The Israeli Defence Forces (IDF) administer a heattolerance test to all HS survivors (20). The test is performed 6-8 weeks post injury and the $\sim 5 \%$ who fail the first iteration are tested again 2-4 weeks later. A second failure results in discharge. British personnel who are hospitalised following HI are referred for physiological testing at the Institute of Naval Medicine (INM) (1). Those considered heat intolerant are medically discharged (personal communication, Headquarters Army Training and Recruiting Agency).

Information on the long-term health implications following an episode of $\mathrm{HI}$ is limited. A four-year follow-up study of 922 USMC personnel who suffered an episode of HI during recruit training provides useful information (21). When compared with appropriately matched controls, HI cases were found to have lower military retention rates $(24 \%$ vs $31 \%$ at four years, respectively), although methodological problems with the study may have exaggerated any difference. Cases also had higher hospitalisation rates (1.4 times that of controls) in the ICD-9 (International Classification of Diseases) categories of "congenital, ill-defined, respiratory, skin, infections and mental diagnoses." The authors were unable to explain why these particular illnesses appear linked to HI. It was found that the association between HI and subsequent hospitalisation decreased over time. Subsequent hospitalisation for HI or rhabdomyolysis was rare, but more 
common in $\mathrm{HI}$ cases, there being too few recurrences of HI (seven of 922 original cases) to provide reliable figures for comparison. Of the 922 cases, 50 had required in-patient treatment, of which half had clinical HS (delirium, obtundation, coma or prolonged amnesia). However, other severe cases of $\mathrm{HI}$ were unavailable to followup owing to a lack of retention, and the authors could not comment upon subsequent susceptibility for these most severe HS cases.

The risks of military training for those with sickle cell trait (SCT) have been investigated by Kark et al (22). Eleven to thirty deaths were observed in SCT recruits per 100,000 recruit training cycles in contrast to one death per 100,000 training cycles for those without SCT. It was suggested that maintaining fluid intake was more important for individuals with SCT. Gardner et al analysed the increased risk and found it significant for heat stroke and sudden cardiac death, but greatest for rhabdomyolysis (200fold increase) (23). It was found that SCT recruits have an overall risk of exerciserelated death 40 times higher than those without. Despite this, it has been concluded that the attributable risk of a military career for those with uncomplicated SCT is sufficiently low that there are no grounds for exclusion (14). Furthermore, little or no excess mortality has been shown for SCT personnel after graduation from basic military training (22).

Malignant hyperthermia $(\mathrm{MH})$ is a pharmocogenetic disorder of skeletal muscle normally triggered by suxomethonium or volatile anaesthetic agents (e.g. enflurane, isoflurane halothane). Administration of these drugs causes a rise in myoplasmic calcium ion concentration which leads to muscle rigidity, hypermetabolism (pyrexia, hypoxia and metabolic acidosis), and sarcolemmal disruption (hyperkalaemia, myoglobinaemia) (24). $\mathrm{MH}$ is diagnosed by in-vitro muscle contracture tests (IVCT) to halothane and caffeine (25). Studies of HS victims have identified some predisposed to MH (26,27). Regardless of the possible connection to $\mathrm{HI}, \mathrm{MH}$ is sufficiently rare that only one officer cadet will have entered the Royal Military Academy Sandhurst (RMAS) since the end of the Second World War possessing the defective gene (6). Further investigation of HS victims has suggested an underlying skeletal muscle abnormality distinct from $\mathrm{MH}$, but involving a similar deregulation of myoplasmic calcium ion concentration $(27,28)$. Bendahan et al used ${ }^{31} \mathrm{P}$ magnetic resonance spectroscopy (MRS) and IVCT to investigate twenty-six French commandos who had experienced HS (28). The subjects displayed abnormal muscle metabolism with similarities to $\mathrm{MH}$ when compared to non-MH controls. They exhibited muscle contracture to halothane and caffeine at a lower level than that which would lead to a diagnosis of $\mathrm{MH}$. On exercise, MRS revealed an early intracellular acidosis that was linked to abnormally high activation of glycogenolysis. A finding of uncertain significance was that the commandos had significantly lower levels of phosphomonoesters at rest. There were two sub-groups of HS subjects identified by IVCT. Contracture to halothane was similar in both groups. However, nineteen subjects exhibited caffeine-induced contracture twice as large as the remaining seven subjects in conjunction with significantly earlier onset of contracture to ryanodine. It was concluded that HS was related to a failure of calcium regulation associated with a latent myopathy that was readily identified by non-invasive MRS. The researchers obtained their subjects from 250 HS cases evaluated with IVCT and MRS, of which 32\% were diagnosed as susceptible to malignant hyperthermia.

Wappler et al investigated 12 subjects with exertion-induced rhabdomyolysis for their susceptibility to $\mathrm{MH}$ (27). The subjects were aged from 12 to 45 years and had been participating in a variety of different physical activities. The controls were a group of older patients undergoing hip replacement. Only one subject had normal IVCT response to halothane and caffeine. Another subject had an equivocal response. The remaining ten all produced an abnormal halothane-caffeine response diagnostic of $\mathrm{MH}$. These subjects also displayed an accelerated and more intense contracture response to ryanodine compared to the controls and non- $\mathrm{MH}$ cases. Genetic analysis showed that three of the subjects had genetic mutations characteristic of $\mathrm{MH}$ at the ryanodine receptor gene. The authors recommended performing muscle biopsy, IVCT and genetic screening on all patients where there was clinical suspicion of exercise-related $\mathrm{MH}$.

A different muscle abnormality was implicated in a non-MH patient who showed profound elevation of CPK 11 days post HS. $\mathrm{He}$ was successfully treated with systemic steroids, later undergoing MRS that suggested a metabolic disorder of skeletal muscle preventing the re-conversion of lactate to pyruvate (29).

There is other muscle pathology that has been associated with HI. Hsu et al concluded that a high proportion of type II muscle fibre increases your risk of HS (30). Muscle biopsy was performed on 37 Taiwanese HS patients to reveal a predominance of type II fibres. These were linked to poorer work efficiency resulting in increased blood lactate thresholds. It was also found that subjects who had developed rhabdomyolysis as a complication of HS had the highest proportion of type II fibres. Blood lactate threshold was suggested as a good indicator for the endurance capacity of recruits before 
training or for patients recovering from HS. It has been suggested that such tests would be more sensitive at detecting heat intolerance than the traditional indices of rectal temperature and heart rate monitored during standard exercise-heat tests (31).

Dehydration has long been considered a significant factor in the development of HI. The concept that "water discipline" would enable servicemen to reduce their physiological need for water has been consigned to history. There is great emphasis within military command that fluid replacement prevents heat casualties $(1,32)$. In recent years some have suggested that water intake is largely irrelevant (6) in cases of HI within the British Army. It should be remembered that the effect of dehydration is not mediated through the cessation of sweating, as most HS cases sweat profusely, but rather through circulatory failure (20). Dehydration was identified as a major cause in $16 \%$ of cases presented by Epstein et al (13). A case series of seven US Army Reserve soldiers who required hospitalisation to correct electrolyte imbalances caused by over-drinking was published in 1998 (33). The US Army later issued revised guidelines for water consumption in 1999 (32). That same year the death of a US Army recruit from acute water intoxication was published (34). The unfortunate soldier was presumed to be suffering from heat illness and given oral fluids until fatal cerebral and pulmonary oedema developed.

Dehydration due to insufficient drinking, in the presence of unrestricted access to water, has been termed voluntary dehydration (35). People with cystic fibrosis (CF) greatly underestimate their fluid needs during exercise in hot conditions, as a result of their sweat having a high salt content and thus depriving them of the hyperosmolar thirst-trigger (36). It has been suggested that carriers of the CF gene may have marginally saltier sweat, resulting in increased risk of salt-deficiency heat exhaustion compared to non-carriers (15). Carriage of the CF gene has yet to be proven as contributing to HI. The significance of this possible link has been addressed by Bricknell (37): "The low incidence of heat illness in servicemen combined with the high prevalence of the heterozygote carriers suggests that the heterozygote state is not a significant factor in the development of heat illness. Thus being a heterozygote carrier would have very little positive predictive value for the risk of developing heat illness in the future even if the case control study showed an association. Therefore there would be no justification to restrict the employability of heterozygote servicemen and thus the test is of no value."

\section{Treatment}

Rapid cooling is essential for the cases of HI with an elevated core temperature because the morbidity and mortality arising from HS is directly related to the peak temperature and its duration $(13,38)$. The best means of lowering body temperature is uncertain and the subject of great debate (39-42). USMC recruits who fall victim to HS are cooled with ice water immersion and skin massage (See Table 2). This approach successfully treated 252 casualties with no fatalities over the period 1975-1990 (43). The use of ice has been criticised for multiple reasons: vasoconstriction leading to increased core temperature, induction of shivering increasing heat production at skin temperatures below $28^{\circ} \mathrm{C}$, discomfort to patient and medical assistants, difficulty in performing cardiopulmonary resuscitation (CPR) and monitoring vital signs, unhygienic conditions if vomiting and diarrhoea occur $(40,41,44)$. Ice water immersion results in the fastest cooling rate $(38,44)$ and provides cardiovascular benefit from the assistance which hydrostatic pressure offers to the hypotensive patient in the form of increased venous return (43). Body cooling units (BCUs) spray a mist of water over the patient and cool by evaporation and convection (44). They can be improvised with shower spray and fan (39). The ideal way to resolve debate would be to conduct a clinical trial comparing such treatment modalities as ice water immersion, tepid water immersion, body cooling units, wet sponges and fans at a suitable HS centre (43). More invasive methods to lower temperature include intravenous or intraperitoneal administration of cool fluid, gastric lavage, enemas and extra-corporeal circulation (45). Military equipment has provided innovative adjuncts to these traditional therapies: inflatable life-rafts filled with water (46), helicopter rotor blade downdraft (43) and evacuation in swift open vehicles. Clear instructions are issued to all Directing Staff (DS) on the treatment of HS in the field (1). However, the Armed Forces have been accused of failing to initiate cooling procedures (6).

Blood Purification Therapy (BPT) was investigated in a group of five classical heat stroke patients by Ikeda and colleagues (49). The three patients given BPT in conjunction with conventional treatment survived, whilst the two patients given conventional treatment alone both died. It was concluded that BPT may be beneficial in the treatment of HS due to the removal of proinflammatory cytokines.

Dantrolene Sodium acts on skeletal muscle to decrease calcium efflux from the sarcoplasmic reticulum, decreasing muscle tone and metabolic heat production (50). It is used in the prophylactic and acute treatment of malignant hyperthermia and neuroleptic malignant syndrome. Two clinical trials have investigated the role of dantrolene in the treatment of classical HS. 
Channa et al administered dantrolene or placebo to twenty classical heat stroke patients and found that dantrolene cooled patients significantly quicker than placebo, but to no clinical benefit (51). Bouchama et al conducted a larger trial involving fifty two classical heat stroke patients (52). There was no decrease in cooling time with dantrolene $(2 \mathrm{mg} / \mathrm{kg}$ iv) and it was concluded that it was not beneficial in the routine treatment of HS.

Opioids have thermoregulatory properties, evidenced by $\mathrm{HS}$ victims with four-fold increases in ß-endorphin/ß-lipotropin (53). Naloxone has been shown to prevent hyperthermia-induced convulsions in rat pups (54) and increase survival times for rats exposed to heat (55). Romanovsky et al found that naltrexone (a wide spectrum opioid receptor antagonist) administered to lab guinea pigs at the onset of HS reduced mortality from three of six controls to zero of five treated (56). Although this was not statistically significant $(p=0.18)$ the authors regarded it as meriting further investigation.

Table 2. Current Cooling Methods for Exertional Heat Stroke at the United States Marine Corps Training Base at Parris Island ${ }^{\star}$

Gaffin SL, Gardner JW, Flinn, SD. Cooling Methods for Heat Stroke Victims. Ann Intern Med 2000; 132 (8): 678.

1. In the field, medical personnel, transportation, and an ice chest are constantly available during physical training. The clinic routinely maintains a dedicated room with two bathtubs full of cold water and ice.

2. In the field, the blouse and pants are removed from a patient who has collapsed from suspected heat stroke; shorts and T-shirt are left on. Rectal temperature is measured; if $>39.4^{\circ}$, the patient is wet down, ice is packed around the groin/axillary areas, and the patient is immediately transported to the clinic on a stretcher. Upon arrival, the stretcher is placed on top of the iced bathtub above the water and ice, with the carrying handles sticking out both ends.

3. Mental status and other vital signs are assessed and blood is drawn for laboratory analyses.

4. $1 \mathrm{~L}$ of normal saline is administered as a bolus.

5. Sheets are dipped into the tub's icy water and are used to cover and drench the patient. Copious ice is added to the top of the sheet to cool the patient further, and the skin is massaged to improve skin blood flow. The sheets are frequently re-wetted with the icy water.

6. Concurrently, the head is constantly irrigated with more ice water, and a fan is directed at the patient.

The patient is not routinely immersed in the ice water in case cardiopulmonary resuscitation becomes necessary. However, if rectal temperature is not reduced sufficiently, the patient is immersed directly into the ice and water. With the above procedure, rectal temperature usually decreases to $39.5^{\circ} \mathrm{C}$ within 15-20 minutes. The patient is removed from the stretcher, rinsed, placed on a gurney and intravenous fluids and laboratory studies are reviewed.
* These victims are young men and women who are otherwise healthy. Victims of classic heat stroke are usually elderly and have concurrent diseases. These factors must be considered; for example, volume therapy should be more conservative - a 250 to $500 \mathrm{~mL}$ bolus, then additional fluid titrated according to response.

\section{Discussion}

Prevention

The study by Gardner et al presents a simple screening test to identify those at risk of $\mathrm{HI}$ (10). High-risk personnel are identified with a $\mathrm{BMI} \geq 22$ and a 1.5 mile run-time $\geq 12 \mathrm{~min}$. Recruits could be identified at the beginning of training and prohibited from participating in strenuous activity until they have improved fitness and lost weight. Given that the incidence of HI decreases significantly on graduation from basic training (22), should such restrictions be extended to serving personnel? The goal of regulations should be the prevention of the most serious cases (resulting in death or long-term disability). A case analysis could be performed on all hospital admissions arising from $\mathrm{HI}$ within the British Army in recent years. Medical records would reveal $\mathrm{BMI}$ and 1.5 mile run times are recorded annually as per Army Training Directives. Use of suitably matched controls would indicate the likely preventative value of implementing such a restrictive policy to all soldiers.

Once the decision of who to screen has been taken, the next question is from what training should this high-risk population be excluded? It has been suggested that personnel above a certain BMI should be barred from performing the Advanced Combat Fitness Test (ACFT) (personal communication, Lt Col Bricknell, 18 August 2000). However, Chung et al were unable to isolate $\mathrm{HI}$ to a particular type of training in their 1996 case series of Taiwanese soldiers (57). Although ACFTs were responsible for $\sim 10 \%$ of casualties in a case series from the British Army, 50\% were participating in military runs, with the remainder engaged in marches, military exercises and other activities at the time of collapse (3). This ties in with American research showing that running imposes a much higher metabolic heat load compared to marching with a heavy load (1000-1200 Watts (W) compared to $500 \mathrm{~W}$, respectively) (14). Better evidence that such screening would lead to a reduction in the incidence of $\mathrm{HI}$ should be sought before resorting to medical downgrading. It would be interesting to know the size of the "high-risk population" in the British Army. Gardner et al identified $20 \%$ of USMC recruits as high risk according to their criteria of BMI and 1.5 mile run-time (10). It would seem reasonable to assume a smaller highrisk population in British recruits given the higher incidence of obesity in the American population as a whole. If screening were 
extended beyond recruit training to all ranks, the observed association of weight gain with length of service could result in large numbers of personnel being banned from performing mandatory military skills such as the ACFT.

Recent research suggests congenital abnormalities of muscle metabolism may be the cause of some cases of HI. True malignant hyperthermia $(\mathrm{MH})$ is rare and may lead to a minority of cases of HS (6). There is evidence for a related condition with a similar dysregulation of calcium cycling (26-28). This may be a factor behind an unknown number of $\mathrm{HI}$ cases. A predominance of type II muscle fibres has also been associated with increased risk of heat illness (30). A large scale cohort study incorporating muscle biopsy (for histology and fibre type analysis) and IVCT (to caffeine, halothane and ryanodine) on entrants to RMAS may provide useful information. A more realistic approach may be a cohort study of blood lactate thresholds, which has been suggested as a possible screening tool for $\mathrm{HI}$ in the future (31).

It is recognised as a commander's responsibility to ensure adequate water is drunk before, during and after strenuous activity and tables with minimum fluid requirements are provided (1). Although dehydration is still a factor in many cases of $\mathrm{HI}$, the adverse effects of excess water consumption should be noted $(33,34)$.

Screening for carriage of the cystic fibrosis gene as a means of reducing heat casualties is unlikely to be worthwhile (37). However, there are four main genetic defects implicated in the aetiology of CF (58). It remains possible that an increased risk of $\mathrm{HI}$ will be associated with one or more of these sub-types. Further case-control studies may shed further light on this hypothesis.

\section{Management}

The immediate treatment of $\mathrm{HI}$ is the rapid reduction of body temperature by any available means. There has been debate over the best cooling methods and a clinical trial at a HS centre would be the obvious way forward (43). With only around one hundred serious cases of $\mathrm{HI}$ occurring annually across the entire British Army (1) it would be difficult to conduct such a study in the UK.

Routine administration of dantrolene sodium to HS patients is not supported by the available evidence $(51,52)$. This research, however, has been conducted with classical HS victims. Further clinical trials with exertional HS patients would be worthwhile. Such studies would prove difficult within the British Army owing to the already low incidence of HS. The usual indications are syndromes characterised by muscle rigidity. Given that most HS victims present with flaccidity, the underlying pathophysiology is probably unrelated. The use of dantrolene sodium may be appropriate for severe HS cases unresponsive to initial treatment and exhibiting muscle rigidity.

Opioid receptor antagonists have shown therapeutic potential in animal models of HS (54-56) and could be investigated further with appropriate human studies.

The management of HS cases wishing to return to duty is difficult, especially as the recovery period appears highly variable $(17,18)$. A subsequent episode, possibly fatal, will expose the services to understandable criticism (6), whilst the rights of healthy, motivated personnel to continue their employment must be protected (4). There are many protocols for evaluating physiological response to exercise in the heat $(9,17,18,57)$. Further research is needed to define what constitutes heat tolerance more precisely. Physiological heat-chamber tests are presently used for the investigation of serious (i.e. hospitalised) cases of HI. It is not known whether such testing would be a suitable routine screening tool. There will be a spectrum of tolerance to exercise-heat tests. Newer protocols measuring blood-lactate thresholds may be sufficiently sensitive to delineate sub-groups of individuals classified heat tolerant with traditional tests (31). A cohort of officer cadets could undergo various protocols on enrolling at RMAS.

The relationship between muscle metabolism abnormalities and HI is poorly understood. It is recommended that all severe HS cases undergo muscle biopsy for histology and fibre type analysis in conjunction with IVCT for abnormal responses to halothane, caffeine and ryanodine. Genetic analysis should also be considered.

\section{Conclusions}

The aetiology of HI is multifactorial, the human body showing a range of tolerance to exercise in the heat. External factors such as environmental heat load, dress, hydration, food and acclimatisation have been the subject of much research. Validated regulations exist to assess and manage these risks to protect the majority of personnel from becoming a heat casualty $(1,9)$. There is a simple screening protocol to identify those at risk of $\mathrm{HI}$ that could be performed at the outset of recruit training. Consideration should be given to excluding such personnel from known high-risk activities. More information as to the implications of implementing such a policy could be obtained from a case-control study of previous HI casualties. The mainstay of treatment continues to be prompt cooling initiated in the field and continued at hospital. Most patients will benefit from intravenous fluids as a means of lowering body temperature and stimulating diuresis, but not all are dehydrated. Despite effective risk management, $\mathrm{HI}$ will continue to occur 
through occasional idiosyncratic thermoregulatory responses to severe exercise. Cases resulting in hospitalisation should be thoroughly investigated to rule out congenital muscle pathology. A similar protocol is recommended on a suitable cohort of RMAS or Airborne Forces entrants in order to elucidate abnormal muscle physiology associated with HI.

\section{Acknowledgments}

We would like to thank the following: Col (Retd) Tony Willman (RAMC Officer Recruiting) for assistance in the early stages of this project. The Royal Defence Medical College Library. Ms Katja Kiessling for reading the manuscript.

\section{References}

1. Heat Illness in the Armed Forces: Prevention and Treatment. Defence Council Instruction, Joint Service Publication 122/2001 HMSO 2001.

2. Burgess JE. Analysis of Y Listings and Medical Discharges of Officer Cadets at RMAS from January 1994 to May 1997, with Actions to Prevent Injuries. F R Army Med Corps 1998; 144: 152-155.

3. Bricknell MCM. Heat Illness: a comparison between UK and Cyprus reports. F R Army Med Corps 1996; 142: 59-61.

4. Dickinson JG. Heat Illness in the Services. F R Army Med Corps 1994; 140: 7-12.

5. Dickinson JG. Heat-exercise hyperpyrexia. $f(R$ Army Med Corps 1989; 135: 27-29.

6. Porter AM. Death of a British Officer Cadet from Heat Illness. Lancet 2000; 355: 569-571.

7. Bricknell MCM. Heat Illness - A Review of Military Experience (Part 2). F R Army Med Corps 1996; 142: 34-42.

8. Bricknell MCM. Heat Illness - A Review of Military Experience (Part 1). F R Army Med Corps 1995; 141: 157-166.

9. Bricknell MCM. Setting heat stress limits for acclimatised soldiers exercising in heat. $\mathcal{F} R$ Army Med Corps 1997; 143: 44-48.

10. Gardner JW, Kark JA, Karnei K, et al. Risk factors predicting exertional heat illness in male Marine Corps recruits. Med Sci Sports Exerc 1996; 28 (8): 939-944.

11. Cooper JK. Preventing Heat Injury: Military Versus Civilian Perspective. Milit Med 1997; 162 (1): 55-58.

12. Hubbard RW. An introduction: the role of exercise in the etiology of exertional heatstroke. Med Sci Sports Exerc 1990; 22 (1): 2-5.

13. Epstein Y, Moran DS, Shapiro Y, Sohar E, Shemar, J. Exertional heat stroke: a case series. Med Sci Sports Exerc 1999; 31 (2): 224-228.

14. Kark JA, Burr PQ, Wenger CB, et al. Exertional Heat Illness in Marine Corps Recruit Training. Aviat Space Environ Med 1996; 67: 354-360.

15. Howarth PJN. The Biochemistry of Heat Illness. $\mathcal{F} R$ Army Med Corps 1995; 141: 40-41.

16. Bendahan D, Kozak-Ribbens G, Rodet L, et al. Characterisation of muscular metabolic abnormalities in malignant hyperthermia subjects: application to diagnosis. Anaesthesiology 1998; 88: 96-107.

17. Shapiro Y, Magazanik A, Udassin R, Ben-Baruch G, Shvartz E, Shoenfeld Y. Heat Intolerance in Former Heatstroke Patients. Ann Intern Med 1979; 90: 913916.

18. Armstrong LE, de Luca JP. Hubbard RW. Time course of recovery and heat acclimation ability of prior exertional heatstroke patients. Med Sci Sports Exerc 1990; 22 (1): 36-48.

19. Chung NK, Shabbir M, Lim CL. Cytokine Levels in Patients with Previous Heatstroke under Heat Stress. Milit Med 1999; 164 (4): 306-310.
20. Shani Y, Moran DS, Heled Y, Shapiro Y, Epstein Y Exertional heat illness [Correspondence]. Lancet 2000; 355 (9219): 1992-1993.

21. Phinney LT, Gardner JW, Kark JA, Wenger CB. Long-term follow-up after exertional heat illness during recruit training. Med. Sci. Sports Exerc 2001; 33 (9): 1443-1448.

22. Kark JA, Ward FT. Exercise and Hemoglobin S. In: Seminars in Hematology 1994; 31 (3): 181-225.

23. Gardner JW, Kark JA. Fatal Rhabdomyolysis Presenting as Mild Heat Illness in Military Training. Milit Med 1994; 159 (2): 160-163.

24. Yaqub B, Aldeeb S. Heat strokes: aetiopathogenesis, neurological characteristics, treatment and outcome. F Neurol Sci 1998; 156: 144-51.

25. The European MH Group. A protocol for the investigation of malignant hyperpyrexia $(\mathrm{MH})$ susceptibility. Br f Anaesth 1984; 56: 1267-1269.

26. Kochling A, Wappler F, Winkler G, Schulte AM, Esch JS. Rhabdomyolysis following severe physical exercise in a patient with predisposition to malignant hyperthermia. Anaesthesia \& Intensive Care 1998; 26 (3): 315-318.

27. Wappler F, Fiege M, Steinfath $M$ et al. Evidence for Susceptibility to Malignant Hyperthermia in Patients with Exercise-induced Rhabdomyolysis. Anaestesiology 2001; 94: 95-100.

28. Bendahan et al. A Non-invasive investigation of Muscle Energetics Supports Similarities Between Exertional Heat Stroke and Malignant Hyperthermia. Anesth Analg 2001; 93: 683-9.

29. Brown J, Mitchell S. A Complicated Case of Exertional Heat Stroke in a Military Setting with Persistent Elevation of Creatine Phosphokinase. Milit Med 1992; 2: 101-103.

30. Hsu YD, Lee WH, Chang MK, et al. Blood lactate threshold and type II fibre predominance in patients with exertional heatstroke. $f$ Neurol Neurosurg Psychiatry 1997; 62: 182-187.

31. Epstein Y. Predominance of type II fibres in exertional heat stroke. Lancet 1997; 350 (9071): 8384.

32. Montain SJ, Latzka WA, Sawka MN. Fluid replacement recommendations for training in hot weather. Milit Med 1999; 164 (7): 512-508.

33. Reynolds MC, Feighery S, Schumaker HD. Complications of Fluid Overload in Heat Casualty Prevention during Field Training. Milit Med 1998; 163 (11): 789-791

34. Garigan TP, Ristedt DE. Death from hyponatraemia as a result of acute water intoxication in an Army basic trainee. Milit Med 1999; 164 (3): 234-8.

35. Rotsthein A, Adolph EF, Wills JH. Voluntary dehydration. In: Adolph EF, ed. Physiology of man in the desert. New York: Interscience 1947: 251270.

36. Bar-Or O. Blimkie CJ. Hay JA. et al. Voluntary dehydration and heat intolerance in cystic fibrosis. Lancet. 1992; 339 (8795): 696-9.

37. Bricknell MCM. Heat Illness and Cystic Fibrosis. Letter in $\mathcal{F}$ R Army Med Corps 1995; 141: 182-183.

38. Gaffin SL, Gardner JW, Flinn, SD. Cooling Methods for Heatstroke Victims. Ann Intern Med 2000; 132 (8): 678.

39. Ndukwu IM, Dematte JE, O'Mara K. Features and Outcome of Classic Heat Stroke [Letters]. Ann Intern Med 1999; 130 (7): 614-615.

40. Slovis CM. Features and Outcome of Classic Heat Stroke [Letters]. Ann Intern Med 1999; 130 (7): 614.

41. Graham BS. Features and Outcomes of Classic Heat Stroke [Letters]. Ann Intern Medicine 1999; 130 (7): 613-614.

42. Duthie DJR. Heat-related illness. Lancet 1998; 352 (9137): 1329-1330.

43. Costrini A. Emergency treatment of exertional heatstroke and comparison of whole body cooling techniques. Med Sci Sports Exerc 1990; 22 (1): 15-18.

44. Weiner JS, Khogali M. A physiological body-cooling unit for treatment of heat stroke. Lancet 1980; 1 (8167): 507-509. 
45. Simon HB. Current Concepts: Hyperthermia. $N$ Engl F Med 1993; 329 (7): 483-487.

46. Sweeney WB, Krafte-Jacobs B, Hansen W, Saldana M. A Method for Shipboard Treatment of Multiple Heat Casualties. Milit Med 1992; 157 (3): 145-147.

47. Seraj MA, Channa AB, al Harthi SS, et al. Are heat stroke patients fluid depleted? Importance of monitoring central venous pressure as a simple guideline for fluid therapy. Resuscitation 1991; 21 (1): 33-39.

48. Dahmarsh NS, Harthi A, Akhtar J. Invasive evaluation of patients with heat stroke. Chest 1993; 103: 1210-1214.

49. Ikeda Y, Sakemi T, Nishihara G, et al. Efficacy of blood purification therapy for heat stroke presenting rapid progress of multiple organ dysfunction syndrome: a comparison of five cases. Intensive Care Medicine 1999; 25 (3): 315-8

50. Moran D, Epstein Y, Weiner M, et al. Dantrolene and Recovery from Heat Stroke. Aviat Space Environ Med 1999; 70: 987-989.

51. Channa AB, Seraj MA, Saddique AA, et al. Is dantrolene effective in heat stroke patients?. Crit Care Med 1990; 18 (3): 290-292.
52. Bouchama A, Cafege A, Devol EB, et al. Ineffectiveness of dantrolene sodium in the treatment of heatstroke. Crit Care Med 1991; 19 (2): 176-180.

53. Appenzeller O, Khoghali M, Carr DB, et al. Makkah Haji: heat stroke and endocrine responses. Ann. Sports Med. 1986; 3: 30-32.

54. Puig MM, Miralles F, Laorden L. Naloxone prevents hyperthermia induced convulsions in immature rats. Methods Find Exp Clin. Pharmacol. 1986; 8 (11): 649-653.

55. Panjwani GD, Mustafa MK, Muhailan A, Aneja IS, Owunwanne A. Effect of hyperthermia on somatosensory evoked potentials in anaesthetised rat. Elecroencephalogr Clin Neurophysiol 1991; 80: 384-391.

56. Romanovsky AA. Blatteis CM. Heat stroke: opioidmediated mechanisms. Fournal of Applied Physiology 1996; 81 (6): 2565-70.

57. Chun NK, Pin CHP. Obesity and the Occurrence of Heat Disorders. Milit Med 1996; 161 (12): 739-742.

58. Davies RJ. 2000 Respiratory Disease. In: Kumar P and Clark M eds. Clinical Medicine. 4th Edition, W B Sanders. 